\author{
Military Technical College \\ Kobry El-Kobbah, \\ Cairo, Egypt
}

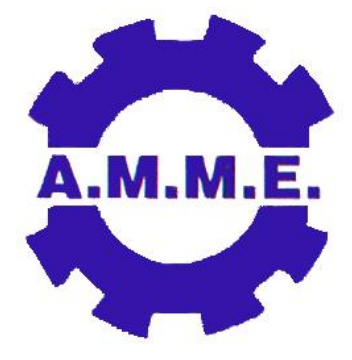

$14^{\text {th }}$ International Conference on Applied Mechanics and Mechanical Engineering.

\title{
Approximate Models for Crashworthiness Design Optimization
}

By

\author{
Hesham Ibrahim * \\ Ramin Sedaghati **
}

\begin{abstract}
:
Design optimization for crashworthiness improvement is one of the most difficult to solve engineering design problems. The difficulty stems from the extremely high computational cost associated with the highly nonlinear finite element models used for simulating the sophisticated crash behaviour of structural components. This paper presents a practical and an efficient methodology for solving such problems. The methodology combines nonlinear finite element analysis, response surface method, design of experiments and numerical optimization. The methodology is applied to improve the crashworthiness performance of the S-rail, which plays a very important role in absorbing most of the impact energy in frontal collisions. The results demonstrate the capability and potential of this methodology in optimizing the crashworthiness design of vehicle structures.
\end{abstract}

\section{Keywords:}

Crashworthiness; Vehicle design; Optimization; LS-DYNA; Automobile structures; Approximate methods; RSM; DOE 
* Egyptian Armed Forces

** Department of Mechanical \& Industrial Engineering, Concordia University, Montreal, Quebec, Canada

\section{Introduction:}

Safety is an important factor in current automobile design due to governmental regulations and increasing consumer awareness. Crashworthiness is the measure of vehicle's safety and thus design for crashworthiness is one of the most important steps in vehicle design cycle. Crashworthiness is typically evaluated numerically using the Nonlinear Finite Element (NFE) method at early and final stages of the design due to the high cost associated with the experimental testing. However, crash analysis of a large-scale problem such as automobile structures is a complex and highly nonlinear problem requiring enormous computational cost e.g. crash analysis of a full frontal impact can extend for more than half a day even with current computational capabilities [1]. On the other hand, weight reduction is another important factor in vehicle design as it directly affects the amount of fuel consumption and can also adversely affect the vehicle's safety. Considering this, crashworthiness design optimization is essential to reach a solution that can optimally satisfy both objectives of safety and weight, and yet meet the required design constraints. Due to inherent significantly high computational costs, and noisy behaviour of objective and constraint functions, crashworthiness design optimization of large-scale problems using traditional gradient based optimization techniques is extremely difficult and may render inaccurate result due to unavailability of the accurate sensitivity analysis. To overcome this problem, approximation techniques are used to create simpler surrogate models instead of the computationally expensive NFE models. The Response Surface Method (RSM) combined with the Design of Experiment (DOE) has been used effectively for solving various engineering problems [2-8]. This paper presents a practical and efficient methodology for solving complicated nonlinear dynamic problems. The methodology is applied to the design improvement of a vehicle S-rail. As shown in Figure 1 the methodology starts by creating an approximate model of the high fidelity nonlinear finite element model. The approximate model is then used to formulate an optimization problem in which the objective is to improve the crashworthiness performance without violating any of the imposed design constraints. Finally, nonlinear finite element simulation is conducted at the resulting optimum design values. This final step is essential to verify that the obtained design is truly an optimum design since the optimization problem is based on approximate models. 


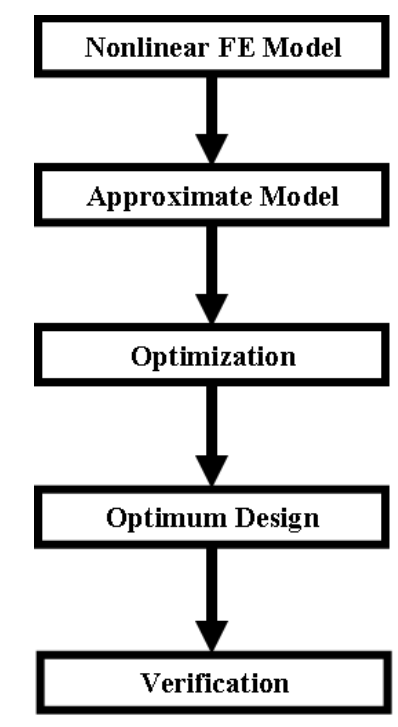

Figure (1): A flow chart of the proposed methodology

\section{Response Surface Method (RSM):}

RSM is a global approximation technique which can be used to construct simple and easy to calculate surrogate models of the highly complex nonlinear FE models. The output models are in the form of smooth polynomial functions describing the output response in terms of the input design variables. Thus the noisy behaviour of output responses which typically encountered in crash analysis problems can be easily alleviated. These polynomials can then be used in evaluating the system response within the design space at an affordable computational cost. However, to create these approximate models, several evaluations are required to identify the unknown polynomial coefficients. Because crashworthiness evaluations are expensive, DOE is used to minimize the number of points at which simulation will take place. Simulations are then performed at these strategic points, and data is processed by regression analysis to identify the unknown polynomial coefficients. The accuracy of the models is quantified to determine their validity to represent the system instead of the true (expensive) models. Having these models validated, they can be effectively used to perform design optimization at a much lower computational cost.

\subsection{Regression Analysis:}

Regression analysis is used to identify the unknown polynomial coefficients in RSM. Let us assume $y=X \beta+\varepsilon$ where $\mathrm{y}$ is an $(n \times 1)$ vector that contains the exact responses calculated from NFE simulations, $X$ is an $(n \times p)$ design matrix determined based on the type of choice of the polynomial functions; $\beta$ is a $(p \times 1)$ vector of the unknown model polynomial coefficients, and $\varepsilon$ is a $(p \times 1)$ vector that contains the errors between exact and approximate responses. Using least squares method, the coefficients can be calculated as:

$$
\beta=\left(X^{T} X\right)^{-1} X^{T} y
$$

\subsection{Approximate Models:}

There are four types of regression models, which can be described as follows: 
(1) A linear model (Model 1) that includes only basic variables and does not include any interaction between variables which is described as:

$$
\hat{y}=a_{0}+a_{1} x_{1}+a_{2} x_{2}+a_{3} x_{3}+a_{4} x_{4}+a_{5} x_{5}
$$

\section{Where:}

$\hat{y}$ is the approximated response

$a_{i}$ are the unknown coefficients of the approximate models

$x_{i}$ are the design variables, which are considered here as the thickness of each part.

This type of model, represent a hyper-plane in the 5 dimension design space with no curvature since only first degree variables are included. The constant term represents the initial value of the response.

(2) A linear model with interaction (Model 2) that includes constant, linear, and interaction terms as in the following form:

$$
\begin{aligned}
& \hat{y}=a_{0}+a_{1} x_{1}+a_{2} x_{2}+a_{3} x_{3}+a_{4} x_{4}+a_{5} x_{5}+a_{12} x_{1} x_{2}+a_{13} x_{1} x_{3}+ \\
& a_{14} x_{1} x_{4}+a_{15} x_{1} x_{5}+a_{23} x_{2} x_{3}+a_{24} x_{2} x_{4}+a_{25} x_{2} x_{5}+a_{34} x_{3} x_{4} \\
& +a_{35} x_{3} x_{5}+a_{45} x_{4} x_{5}
\end{aligned}
$$

This model allows for the first order interaction between variables and it represents a hyperplane in the variables space.

(3) A quadratic model (Model 3) that includes constant, linear, interaction, and squared terms in the following form:

$$
\begin{aligned}
& \hat{y}=a_{0}+a_{1} x_{1}+a_{2} x_{2}+a_{3} x_{3}+a_{4} x_{4}+a_{5} x_{5}+a_{12} x_{1} x_{2}+a_{13} x_{1} x_{3}+ \\
& a_{14} x_{1} x_{4}+a_{15} x_{1} x_{5}+a_{23} x_{2} x_{3}+a_{24} x_{2} x_{4}+a_{25} x_{2} x_{5}+a_{34} x_{3} x_{4}+ \\
& a_{35} x_{3} x_{5}+a_{45} x_{4} x_{5}+a_{11} x_{1}{ }^{2}+a_{22} x_{2}{ }^{2}+a_{33} x_{3}{ }^{2}+a_{44} x_{4}{ }^{2}+a_{55} x_{5}{ }^{2}
\end{aligned}
$$

This model is quite flexible in representing wide varieties of models especially when there is no prior knowledge about the type of the model to be used. Thus it is the default model of choice in RSM application. It represents a hyper plane in the design space with curvature due to the second degree terms.

(4) A pure quadratic model (Model 4) that includes constant, linear, and squared terms described in the following form:

$$
\hat{y}=a_{0}+a_{1} x_{1}+a_{2} x_{2}+a_{3} x_{3}+a_{4} x_{4}+a_{5} x_{5}+a_{11} x_{1}^{2}+a_{22} x_{2}{ }^{2}+a_{33} x_{3}{ }^{2}+a_{44} x_{4}^{2}+a_{55} x_{5}^{2}
$$

This model represents a hyper-plane with curvature, but it includes no interacting terms. Meta-model selection is the process in which the designer tries to find the best approximation that fits the data. A prior knowledge about the system being modeled will facilitate this process. This knowledge can include the type of variables important to the output response, which will help in reducing the number of variables by screening out important ones. Also, knowledge about the type and level of interaction between variables, if any exists, will help in formulating the approximate model. However, this information is not 
always available, and a trial and error process is inevitable. In such a case, different metamodels are evaluated based on their success in approximating the data; the model with the highest accuracy is then selected.

\subsection{Measuring Accuracy:}

It is important that the accuracy of the developed meta-model is verified so that it can be used with confidence in the optimization process. The coefficient of determination known as $R^{2}$ is typically used to check the model's ability to identify the variation within the output response [9] and is defined as:

$$
R^{2}=1-\frac{S S E}{S S T}
$$

where

$$
S S E=\sum_{i=1}^{n}\left(y_{i}-\hat{y}_{i}\right)^{2}
$$

and

$$
S S T=\sum_{i=1}^{n}\left(y_{i}-\bar{y}_{i}\right)^{2}
$$

Where ${ }^{y_{i}}$ is the true output response, here calculated from nonlinear FE analysis, $\hat{y}_{i}$ is the approximate response calculated from RSM, $\bar{y}$ is the average of the true response, and $n$ is the number of design points used to generate the model. $R^{2}$ varies between 0 and 1 , where values close to 1 mean that the approximate model has high ability to explain the variations within the output response.

It should be noted that the value of $R^{2}$ will not decrease by adding new variables. This means that $R^{2}$ can increase even by adding unnecessary variables. In fact $R^{2}$ can be brought to 1 if a model with $(n-1)$ of variables is used to fit $n$ points. Therefore, $\operatorname{adj} R^{2}$ is used instead, which adjusts $R^{2}$ by dividing both SSE and SST by their associated degrees of freedom as:

$$
\operatorname{adj} R^{2}=1-\frac{\frac{S S E}{(n-p)}}{\frac{S S T}{(n-1)}}
$$

where $p$ is the number of design variables. $\operatorname{adj} R^{2}$ will then account for adding unimportant design variables. Since for $\mathrm{n}$ points, the value of $\frac{S S T}{(n-1)}$ is constant, then $\operatorname{adj}^{2}$ will increase only when adding a new variable results in reducing the value of $\frac{S S E}{(n-p)}$. In fact adjR may 
even decrease when adding a new variable decreases the value of $(n-p)$ more than it decreases the value of SSE.

\section{Design of Experiments:}

To determine the polynomial coefficients $\beta$, simulation is conducted at various points in the design space to generate the $y$ responses. The location of these points should be chosen carefully to generate accurate models. Naturally, as the number of points covering the design space increases, the model's accuracy also increases. However, since crashworthiness simulation is computationally expensive, the number of simulations should be optimized to produce accurate models with minimum simulation runs. DOE is a statistical technique which can be used to locate the optimum number of experiments to be carried on. The D-optimality criterion is one of the most popular techniques which depends on maximizing the value of $\left|X^{T} X\right|_{\text {[9], as the value of the variance in } \beta,}, \operatorname{var}(\beta)$ is related to $\left(X^{T} X\right)$ as:

$$
\operatorname{var}(\beta) \propto\left(X^{T} X\right)^{-1}
$$

Therefore considering Eq.(10), maximizing $\left|X^{T} X\right|$ leads to less variation in $\beta$ and more accurate models.

\section{Optimization of a Vehicle S-Rail:}

The S-Rail is a longitudinal thin walled structure in the shape of the letter $S$ and is used as a supporting structure. The automobile sub systems are supported on lateral cross members which are connected to the S-Rail. The S-Rail and the cross members basically form the chassis frame of the vehicle structure [10]. The S-Rail plays an important role as a crush member, especially in frontal crashes. In this section, the principle of using meta-models for improving the crashworthiness of the S-Rail by mathematical optimization is demonstrated. To lower the computational requirements, a simple prototype FE model is used.

\subsection{Problem Statement:}

It is required to improve the baseline design of an S-Rail for crashworthiness. The objective is to increase the amount of impact energy that this structure can absorb at an impact velocity of $10 \mathrm{~m} / \mathrm{s}(27 \mathrm{~km} / \mathrm{h})$. The baseline design as shown in Figure 2 consists of three parts. The thickness of each part is considered as a design variable, thus there are three design variables. The initial design values are assumed to be $\left[x_{1}=0.5, x_{2}=2.5, x_{3}=2.5\right] \mathrm{mm}$. With these initial values, the S-Rail weighs $3.1 \mathrm{~kg}$ and absorbs a maximum of $251.4 \mathrm{~J}$ at the designated impact speed of $27 \mathrm{~km} / \mathrm{h}$. Lower and upper bounds of the design variables are assumed to be 0.5 and 2.5 , respectively. 


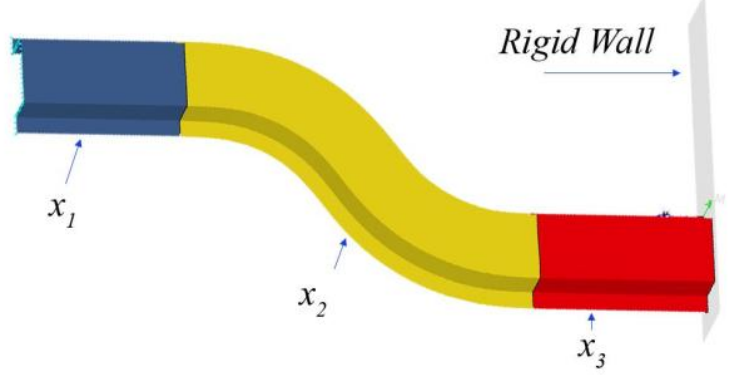

Figure (2): View of the S-Rail

\subsection{Solution Approach and Results:}

One way of solving this problem is to conduct physical experimental tests at every point in the design space until a feasible optimum design is reached. However, considering a step size of 0.5 $\mathrm{mm}$ for each design variable, yields a total of $(5 \text { [levels] })^{3 \text { (variables] }}=125$ design points, which is clearly impractical and very expensive to perform. It should be noted that the number increases to 729 if a step size of $0.25 \mathrm{~mm}$ is used instead. Even if a DOE technique is used to minimize the number of experiments, the time and cost is still inconceivable. Also, using intuition or even experience to guide the tests does not guarantee that a feasible solution can be reached.

On the other hand, adopting numerical simulation by nonlinear FE modeling is an attractive option. Although, the method has not been matured enough to the point that no physical experiments are required, the method is admissibly reliable to cut down on the number of required tests, and mostly, only a final physical test is needed to validate the simulation results. Nevertheless, due to the complexity of nonlinear dynamic problems, FE simulation does come with its own cost in terms of computational demands. Consequently, it is also impractical to simulate the 729 designs or even the 125 ones, especially in the current competitive environment, in which the design time window is being narrowed steadily. For these reasons, a meta-model is established with an appropriate number of simulations by a DOE technique and this meta-model is then used in a systematic form to search for a feasible optimum solution.

\section{The FE Model:}

The FE model has been developed in LS-DYNA to simulate the impact behaviour of the SRail. For the sake of simplicity and lowering the computational cost, a model at a reduced scale has been constructed. A scale of 0.4 is used to scale down the full model, and scaling laws [9] are used to transform other model parameters, such as component mass and initial kinetic energy, into reduced values. The parameters of both the full scale model and the reduced scale model are presented in Table 1. It should be noted that neither material properties nor impact speed can be scaled [11].

Table (1): Full scale and prototype model parameters for the S-Rail

\begin{tabular}{|l|l|l|}
\hline Quantity & Full scale & Prototype \\
\hline Component mass $(\mathrm{kg})$ & 46.8 & 3 \\
\hline
\end{tabular}




\begin{tabular}{|l|l|l|}
\hline Kinetic energy (kJ) & 586 & 3.75 \\
\hline
\end{tabular}

The FE model consists of 5093 shell elements modeled by the Belytschko-Lin-Tsay element (type 2 in LS-DYNA) with an average element length of $6 \mathrm{~mm}$. The MAT PLASTIC KINEMATIC material model (type 24 in LS-DYNA) is used to model the behaviour of material under impact. The material properties are presented in Table 2.

Table (2): Material properties of the S-Rail

\begin{tabular}{|l|l|}
\hline Property & value \\
\hline Density (kg/m ${ }^{3}$ ) & 7820 \\
\hline Young's modulus (GPa) & 207 \\
\hline Poisson's ratio & 0.3 \\
\hline Yield strength (MPa) & 200 \\
\hline $\begin{array}{l}\text { Tangential modulus } \\
\text { (GPa) }\end{array}$ & 0.2 \\
\hline
\end{tabular}

The D-optimal criterion is used to generate data points for building the meta-model. For a design with three variables coupled with a quadratic RSM model, a minimum of 10 points is needed according to $\frac{(n+1) \cdot(n+2)}{2}$ where $n$ is the number of design variables. Redhe et al. [12] have suggested using 1.5 times this number when using the D-optimal criterion. Here, 20 points are used to generate more accurate results. Using design of experiments based on the D-optimal criterion, the design matrix can now be easily established as provided in Table 3.

Table (3): The design matrix of the response surface model for the S-Rail

\begin{tabular}{|c|c|c|c|c|c|c|c|c|c|c|c|c|c|c|c|c|c|c|c|c|}
\hline & 1 & 2 & 3 & 4 & 5 & 6 & 7 & 8 & 9 & 10 & 11 & 12 & 13 & 14 & 15 & 16 & 17 & 18 & 19 & 20 \\
\hline$X_{1}$ & 1 & 3 & 3 & 1 & 2 & 2 & 1 & $\begin{array}{l}0 . \\
9\end{array}$ & $\begin{array}{l}0 . \\
5\end{array}$ & $\begin{array}{l}2 . \\
5\end{array}$ & $\begin{array}{l}2 . \\
3\end{array}$ & $\begin{array}{l}1 . \\
7\end{array}$ & $\begin{array}{l}2 . \\
5\end{array}$ & $\begin{array}{l}2 . \\
5\end{array}$ & $\begin{array}{l}1 . \\
5\end{array}$ & $\begin{array}{l}2 . \\
5\end{array}$ & $\begin{array}{l}0 . \\
5\end{array}$ & $\begin{array}{l}1 . \\
7\end{array}$ & $\begin{array}{l}2 . \\
5\end{array}$ & $\begin{array}{l}1 . \\
5\end{array}$ \\
\hline$X_{2}$ & 1 & 1 & 1 & 3 & 1 & 3 & 1 & $\begin{array}{l}2 . \\
5\end{array}$ & $\begin{array}{l}1 . \\
3\end{array}$ & $\begin{array}{l}0 . \\
5\end{array}$ & $\begin{array}{l}2 . \\
5\end{array}$ & $\begin{array}{l}1 . \\
3\end{array}$ & $\begin{array}{l}2 . \\
5\end{array}$ & $\begin{array}{l}2 . \\
5\end{array}$ & $\begin{array}{l}0 . \\
5\end{array}$ & $\begin{array}{l}2 . \\
3\end{array}$ & $\begin{array}{l}1 . \\
5\end{array}$ & $\begin{array}{l}2 . \\
5\end{array}$ & $\begin{array}{l}1 . \\
1\end{array}$ & $\begin{array}{l}1 . \\
5\end{array}$ \\
\hline$X_{3}$ & 1 & 1 & 1 & 2 & 1 & 3 & 1 & $\begin{array}{l}0 . \\
5\end{array}$ & $\begin{array}{l}2 . \\
5\end{array}$ & $\begin{array}{l}2 . \\
5\end{array}$ & $\begin{array}{l}0 . \\
5\end{array}$ & $\begin{array}{l}1 . \\
7\end{array}$ & $\begin{array}{l}1 . \\
5\end{array}$ & $\begin{array}{l}2 . \\
5\end{array}$ & $\begin{array}{l}2 . \\
5\end{array}$ & $\begin{array}{l}2 . \\
5\end{array}$ & $\begin{array}{l}2 . \\
5\end{array}$ & $\begin{array}{l}0 . \\
5\end{array}$ & $\begin{array}{l}0 . \\
5\end{array}$ & $\begin{array}{l}1 . \\
7\end{array}$ \\
\hline
\end{tabular}

Next, LS-DYNA simulations are performed at each of the design points provided in Table 3 and output responses are evaluated. Since the objective is to maximize the amount of Impact Energy (IE) absorbed without increasing the weight (Mass), these two outputs are then collected and used for building the meta-models.

The four models described in section 2.2 are used to construct the approximate model of the S-Rail. The accuracy of the models is evaluated using the adj $\mathrm{R}^{2}$ and the values are provided in Table 4. The results show that the quadratic model (Model 3 ) has the highest accuracy and thus is used to approximate the output responses. The $\operatorname{adj}^{2}$ for Mass is one for all model 
types because Mass is a function of cross section area, which in turn is a function of parts thicknesses. Thus, the variation within Mass can be totally explained by parts thicknesses.

Table (4): Values for adji ${ }^{2}$ for different RSM models for the S-Rail

\begin{tabular}{|l|l|l|}
\hline & Mass & IE \\
\hline Model 1 & 1 & 0.75 \\
\hline Model 2 & 1 & 0.92 \\
\hline Model 3 & 1 & 0.97 \\
\hline Model 4 & 1 & 0.80 \\
\hline
\end{tabular}

Now, an optimization problem can be formulated based on the design requirements and developed meta-models, which can be used to represent the objective and the constraint functions as follows:

Find $X^{*}$ that :

Maximizes IE

Subject to: Mass-Mass original $\leq 0$

where, $\mathrm{x}_{\mathrm{L}} \leq \mathrm{x} \leq \mathrm{x}_{\mathrm{U}}$

The optimization problem is solved using the Sequential Quadratic Programming (SQP) algorithm [13], which is a powerful gradient based nonlinear mathematical programming technique in MATLAB. After 9 iterations, as shown in Figure 3, the algorithm converged to a feasible optimal solution at $X^{*}=\left(\begin{array}{lll}2 & 2 & 2\end{array}\right)$

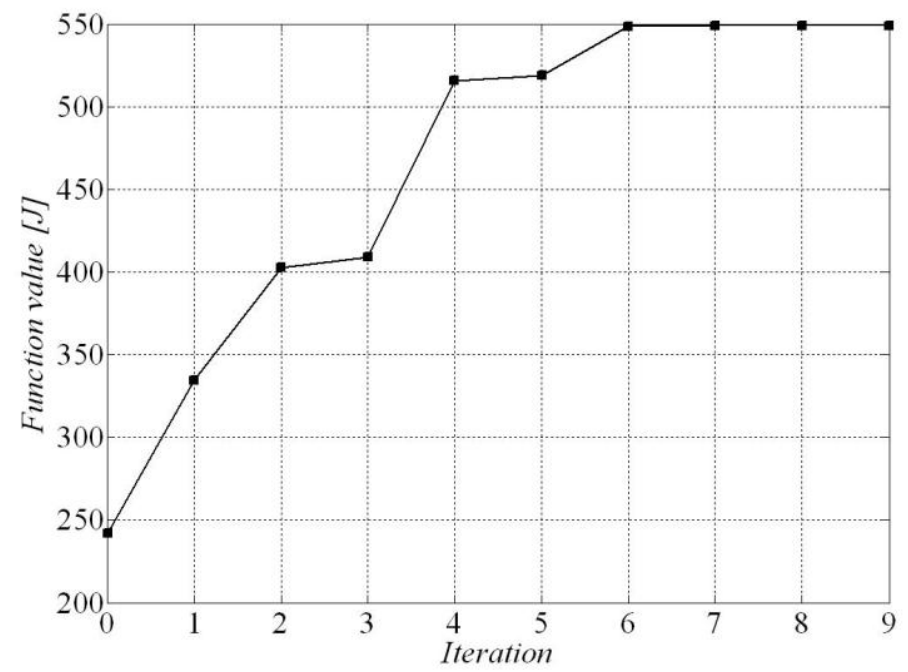

Figure (3): Iteration history of the optimization problem of the S-Rai I

A full FE analysis of the model at this optimal solution yields a value of $528 \mathrm{~J}$ for IE, which is more than double the initial value $(251.4 \mathrm{~J})$. Mass is found to be slightly less than $3 \mathrm{~kg}$, Thus the derived optimal design not only has increased IE appreciably, it has reduced mass slightly as well.

\subsection{Adding Another Constraint to the Problem:}


While inspecting the time response of the Rigid Wall reaction Force (RWF), a peak value (RWFmax) of $60.34 \mathrm{~N}$ is observed which is about 3.4 times the corresponding value in the baseline design. According to the regulations, RWFmax must not exceed $40 \mathrm{~N}$. In fact, the baseline design marked $17.6 \mathrm{~N}$ for RWFmax.

Considering this, another meta-model was established to account for RWFmax. Again, in this case, the quadratic model (Model 3), also proved to be more accurate than other models with a value of 0.95 for the adj $R^{2}$ parameter.

The optimization problem is redefined to reflect the new design requirement by adding another constraint to the optimization problem as:

Find $\mathrm{X} *$ that :

Maximizes IE

Subject to: Mass-Mass original $\leq 0$

RWFmax -RWFlimit $\leq 0$

where, $\mathrm{x}_{\mathrm{L}} \leq \mathrm{X} \leq \mathrm{x}_{\mathrm{U}}$

After 10 iterations, as shown in Figure 4, the algorithm converged to a feasible solution at $\mathrm{X}^{*}$ $=(2.52 .271)$.

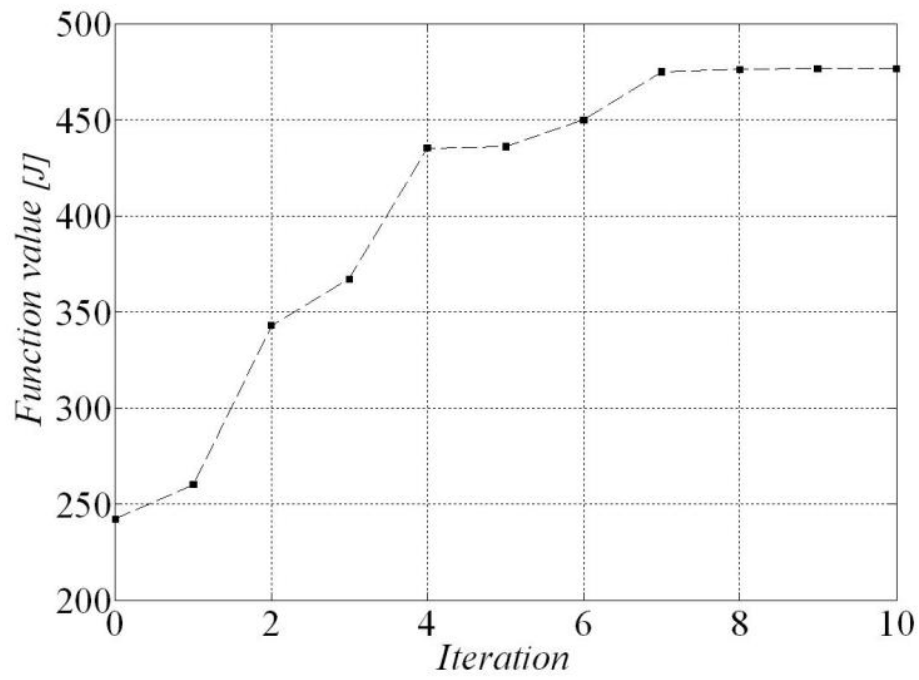

Figure (4): Iteration history of the constrained optimization problem of the S-Rail

This design configuration weighs slightly less than $3 \mathrm{~kg}$ and after conducting full FE simulation on the optimal solution, it gives a value of $522.75 \mathrm{~J}$ for IE and $37 \mathrm{~N}$ for RWFmax. The values for Mass, IE, RWFmax for the optimum design without consideration of RWFmax as a constraint (Design 1) and the optimum design with consideration of RWFmax as a constraint (Design 2) are all normalized with respect to the baseline design and shown in Figure 5. 


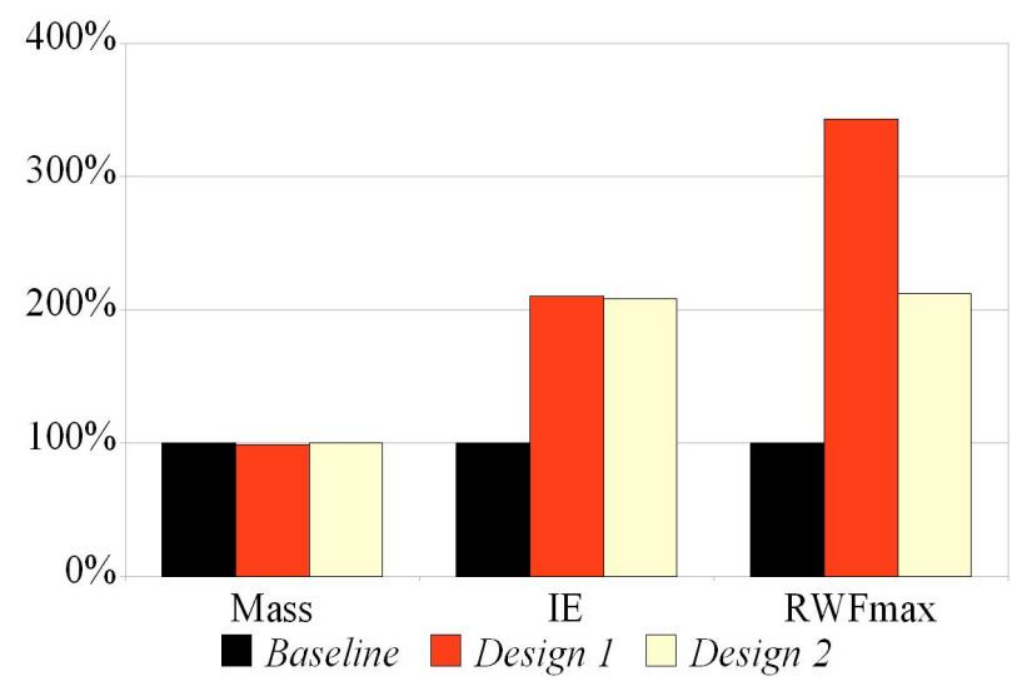

Figure (5): Comparison between Design 1, Design 2 and baseline for Mass, IE and RWFmax Examination of the results reveals that by conducting the design optimization based on RSM on the S-Rail component, one can considerably increase IE of the S-Rail while guarding against maximum allowable rigid wall force. The optimal solutions basically obtained parts' thicknesses for better energy absorption and the two optimal designs improved IE by approximately two-folds while keeping the mass slightly lower than the mass of the baseline design.

\section{Conclusions:}

The complicated nature of the physical crash processes of complex vehicle structures makes the design optimization process for crashworthiness a very challenging task. In this research, a practical and accurate methodology for design optimization of vehicle structures for crashworthiness has been developed. The methodology is based on creating a modified NFE model of the most significant structural component to the total amount of impact energy absorbed. Then, the RSM is used to create an approximate model of the modified NFE model, which can be effectively used in design optimization to improve the crashworthiness performance of the whole vehicle. An illustrative example has been used to demonstrate the methodology.

\section{References:}

[1] S. Natori and Q. Yu. "An Application of CAP (Computer-Aided Principle) to Structural Design for Vehicle Crash Safety". SAE Technical paper 2007-01-0882, 2007.

[2] Hill, William J., and William G. Hunter. "A Review of Response Surface Methodology: A Literature Survey." Technometrics. 8. 4, 571-590, 1966.

[3] Zheng, Y., and P.K. Das. "Improved Response Surface Method and Its Application to Stiffened Plate Reliability Analysis." Engineering Structures. 22. 5, 544-551, 2000.

[4] Mullis, James. Application of Response Surface Method to the Design of an Air Defense Interceptor: A Thesis. Thesis (M.S.E.) University of Alabama in Huntsville, 2005. 
[5] Oraon, B., G. Majumdar, and B. Ghosh. "Application of Response Surface Method for Predicting Electroless Nickel Plating." Materials and Design. 27. 10, 1035-1045, 2006.

[6] Pula, W., and J. Bauer. "Application of the Response Surface Method." courses and lectures- international centre for mechanical sciences. 491, 147-168, 2007.

[7] Fen, C. -S., H. -C. Cheng, and C. -C. Ku. "An effective response surface-based optimisation approach for soil vapour extraction system design." Civil Engineering and Environmental Systems. 25. 3, 227-243, 2008.

[8] Mesbahi, Ehsan, and Yongchang Pu. "Application of ANN-Based Response Surface Method to Prediction of Ultimate Strength of Stiffened Panels." Journal of Structural Engineering. 134. 10, 2008.

[9] D. C. Montgomery, E. A. Peck, and G. G. Vining. Introduction to linear regression analysis. J. Wiley \& Sons, 2006.

[10] J. C. Brown, A. J. Robertson, and S. T. Serpento, Motor Vehicle Structures: Concepts and Fundamentals, SAE International, 2001.

[11] N. Jones, Structural Impact, Cambridge University Press, 1997.

[12] M. Redhe, J. Forsberge, T. Janssone, P. O. Marklund, and L. Nilsson, Using the response surface methodology and the D-optimality criterion in crashworthiness related problems," Structural and Multidisciplinary Optimization 24(3), pp. 185\{194, 2002.

[13] J. S. Arora, Introduction to optimum design, McGraw-Hill, New York, 1989. 